\title{
A BOUNDARY VALUE PROBLEM FOR THIRD-ORDER LINEAR PARTIAL DIFFERENTIAL EQUATIONS OF COMPOSITE TYPE ${ }^{1}$
}

\author{
R. B. DAVIS
}

The third-order partial differential equation

$$
L(u)=b_{1} u_{x x x}+b_{2} u_{x x y}+b_{3} u_{x y y}+b_{4} u_{y y y}+\cdots=0
$$

(where $b_{1}, b_{2}, b_{3}$, and $b_{4}$ are functions of $x$ and $y$, subscripts denote partial differentiation, and the dots, as in the sequel, indicate terms involving only lower order derivatives) is called composite $[5, \mathrm{p}$. $136],{ }^{2}$ for a set $G$ of values of $(x, y)$, if the equation

$$
b_{1} p^{3}+b_{2} p^{2} q+b_{3} p q^{2}+b_{4} q^{3}=0
$$

determines exactly one nonrepeated real ratio $p: q$ for all $(x, y) \in G$. The present paper establishes a canonical form for third order partial differential equations of composite type, and using this, proves the existence and uniqueness of the solution to a certain boundary value problem in the linear case.

Composite equations occur in the Euler equations for two-dimensional incompressible flow, and in the linearized theory of compressible sub-sonic flow. Boundary value problems for (1), different from the present one, have been considered in [7] and [8].

1. Reduction to canonical form. Let (1) be composite throughout a bounded open simply-connected region $R$ and let $b_{1}, \cdots, b_{4} \in C^{1}$ in $R$ (by the notation of $f \in C^{n}$, we mean $f$ has continuous $n$ th-order partial derivatives); then (1) can be written

$$
\begin{aligned}
\left(\alpha u_{x x}+\beta u_{x y}+\gamma u_{y y}\right)_{x} A+ & \left(\alpha u_{x x}+\beta u_{x y}+\gamma u_{y y}\right)_{y} B \\
& +c_{1} u_{x x}+c_{2} u_{x y}+c_{3} u_{y y}+\cdots=0
\end{aligned}
$$

(since the system of equations $\alpha A=b_{1}$, etc., is necessarily solvable); moreover, $A$ can be chosen so that $A \neq 0, A \in C^{1}$ in $R$; then $\alpha, \beta, \gamma$, and $B \in C^{1}$ in $R$, and $\beta^{2}-4 \alpha \gamma<0$ there. We may thus make a change of independent variable (see [3, pp. 127-128]) and write (2) in the form

Presented to the Society, February 23, 1952; received by the editors January 14, 1952.

1 This paper is part of a thesis submitted by the author in partial fulfillment of the requirements for a Ph.D., Massachusetts Institute of Technology, Spring, 1951. The author wishes to express his appreciation of suggestions received from Professor Norman Levinson, Professor C. C. Lin, and Dr. F. E. Browder.

2 Numbers in brackets refer to the bibliography at the end of the paper. 


$$
(\Delta u)_{\xi} \tilde{A}+(A u)_{\eta} \tilde{B}+c_{4} u_{\xi \xi}+c_{5} u_{\xi \eta}+c_{6} u_{\eta \eta}+\cdots=0,
$$

where $\Delta=\partial^{2} / \partial \xi^{2}+\partial^{2} / \partial \eta^{2}$.

Now,

$$
(\tilde{A})^{2}+(\tilde{B})^{2}=\left(A \xi_{x}+B \xi_{y}\right)^{2}+\left(A \eta_{x}+B \eta_{y}\right)^{2} \neq 0 \quad \text { in } R
$$

(cf. the array of the Jacobian as the matrix of a nonsingular transformation, and note that $(A, B)$ is a nonzero vector), and $\tilde{A}, \tilde{B} \in C^{1}$ in $R$, so that, from the usual theory of autonomous systems $[1, \mathrm{pp} .78$ ff.] we can solve the equations

$$
d \xi / d z=\tilde{A} \text { and } \quad d \eta / d z=\tilde{B}
$$

for a family of curves $\xi=\xi(z), \eta=\eta(x)$ which never terminate in $R$. The same is true for the orthogonal trajectories, which are solutions of

$$
d \xi / d t=-\tilde{B}, \quad d \eta / d t=\tilde{A} .
$$

We shall call the solutions of (3) z-curves (or characteristics), and those of $\left(3^{\prime}\right) t$-curves. Note that the $t$-curves are the curves $z=$ constant, and vice versa. We have proved:

LEMMA. If (1) is composite at every point of an open bounded simplyconnected region $R$, and if $b_{1}, \cdots, b_{4} \in C^{1}$ on $R$, then by a change to the new independent variables $z$ and $t$, (1) may be reduced to the form

$$
(\Delta u)_{z}+c_{7} u_{z z}+c_{8} u_{z t}+c_{9} u_{t t}+\cdots=0 .
$$

An equivalent form, obtained by rearranging the lower-order terms, is:

$$
\Delta\left(u_{z}\right)+c_{10} u_{z z}+c_{11} u_{z t}+c_{12} u_{t t}+\cdots=0 .
$$

2. Boundary value problem. Let $T$ be a closed subset of $R$, with a rectifiable boundary $W$. Let $W^{\prime} \subset T$ be a curve extending from $W$ to $W$, cutting every $z$-curve in $T$ exactly once and being tangent to none. With $z$ and $t$ defined as before, and with the requirement that the equation of $W^{\prime}$ be $z=W^{\prime}(t)$, where $W^{\prime}(t) \in C^{3}$, we shall prove:

ThEOREM. Let

$$
L(u)=\Delta\left(u_{z}\right)+a u_{2 z}+b u_{z t}+c u_{z}+d u_{t}+e u=f,
$$

and suppose that:

(H1) $a, b, c, d, e$, and $f$ are functions of $z$ and $t$ belonging to $C^{1}$ on $T$;

(H2) the classical Green's function for Laplace's equation, $K(z, t ; r, s)$, for the region $T$, exists and satisfies 


$$
\begin{aligned}
& \left|K_{z}\right|<\frac{\text { constant }}{\left((z-r)^{2}+(t-s)^{2}\right)^{1 / 2}} \\
& \left|K_{t}\right|<\frac{\text { constant }}{\left((z-r)^{2}+(t-s)^{2}\right)^{1 / 2}}
\end{aligned}
$$

on $T$;

(H3) $h$ and $g$ are functions of $z$ and $t$, with $h \in C^{3}$ on $T, g \in C^{3}$ on $W^{\prime}$. Conclusion: Then either the equation

$$
L(u)=\Delta\left(u_{z}\right)+a u_{z z}+b u_{z t}+c u_{z}+d u_{t}+e u=0
$$

admits a solution $u \in C^{3}$ such that $u_{z}=0$ on $W$ and $u=0$ on $W^{\prime}$, with $u \neq 0$ in $T$; or else there exists a unique function $u$ of $z$ and $t$ such that $u \in C^{3}, u$ satisfies $\left(1^{\prime}\right)$, and $u_{z}=h$ on $W, u=g$ on $W^{\prime}$.

(We shall discuss below two cases in which the first alternative is impossible.)

Remark on H2. Precisely this condition is familiar in the usual second-order "method of Levi" (cf. [3, pp. 282-284]). Lichtenstein [6, pp. 1281-1282] has proved that, if the image of $W$ referred to orthogonal $z, t$ axes has continuous curvature, then inequalities (4) are satisfied. This will be true if $B$ has continuous curvatures in $x, y$ space, since $z$ and $t$ are continuously twice-differentiable functions of $x$ and $y$ [3, pp. 127-128].

Remark on the missing term in $u_{t t}$. Equation ( $\left.1^{\prime}\right)$ contains no term in $u_{t t}$. If such a term is present after reduction to canonical form, it must be eliminated in order to apply the present proof. This can be done in the case of constant coefficients by a change of dependent variable of the form $u=P v$, where $P$ is a suitably-chosen known function.

Proof of TheOREM. Hypothesis $\mathrm{H} 3$ permits us to reduce to zero boundary values, by letting $w_{z}=h$ on $T, w=g$ on $W^{\prime}$; then $u_{1}=u-w$ satisfies $L\left(u_{1}\right)=f_{1}$, with unchanged operator $L$, while $\left(u_{1}\right)_{z}=0$ on $W$, and $u_{1}=0$ on $W^{\prime}$. We may thus assume that $h=0$ and $g=0$. As above, let $K(z, t ; r, s)$ be the classical Green's function for Laplace's equation for the region $T[3,239 \mathrm{ff}$.], expressed in $z, t$ coordinates.

Let

$$
H(z, t ; r, s)=-\int_{W^{\prime}(t)}^{z} K(w, t ; r, s) d w
$$

Finally, let

$$
u(z, t)=\iint_{T} H(z, t ; r, s) \phi(r, s) d r d s,
$$


where we shall determine $\phi$ presently. For any $\phi \in C$ on $T$, we may change the order of integration (this is easily justified by separating $K$ into its logarithmic and harmonic parts), and hence we have $u_{3}=0$ on $W, u=0$ on $W^{\prime}$ (cf. [3, pp. 240-241]). If, further, $\phi \in C^{1}$ on $T$, then $u, u_{z}, u_{t}, u_{z z}$, and $\Delta\left(u_{z}\right)$ all exist and are continuous in $T$ $[3$, pp. $228 \mathrm{ff}$.$] , and \Delta\left(u_{z}\right)=-2 \pi \phi$.

We now determine $\phi$ so that either $u$ satisfies $\left(1^{\prime}\right)$, or else $u$ satisfies $\left(1^{\prime \prime}\right)$.

Substituting into $\left(1^{\prime}\right)$, and noting that we may differentiate under the integral sign $[3$, p. 228], and that, in consequence of $\mathrm{H} 2$, the harmonic part of $K$ when differentiated is integrable [3, p. 283], we get

$$
\begin{aligned}
-2 \pi \phi(z, t) & -\iint_{T}\left\{a(z, t) K_{z}(z, t ; r, s)+b K_{t}+c K\right. \\
& +d\left[\int_{W^{\prime}(t)}^{z} K_{t}(w, t ; r, s) d w-K\left(W^{\prime}(t), t ; r, s\right) W_{t}^{\prime}(t)\right] \\
& \left.+e \int_{W^{\prime}(t)}^{z} K(w, t ; r, s) d w\right\} \phi(r, s) d r d s=f(z, t),
\end{aligned}
$$

and hence $u$ will satisfy $\left(1^{\prime}\right)$ if $\phi \in C^{1}$, and if

$$
\phi(z, t)=-(1 / 2 \pi) f(z, t)+\iint_{T} G(z, t ; r, s) \phi(r, s) d r d s
$$

when

$$
G(z, t ; r, s)=a K_{z}+b K_{t}+\cdots+e \int_{W^{\prime}(t)}^{z} K .
$$

Similarly, $u$ will satisfy $\left(1^{\prime \prime}\right)$ if $\phi \in C^{1}$, and if

$$
\phi(z, t)=\iint_{T} G(z, t ; r, s) \phi(r, s) d r d s .
$$

Equation (5) is a Fredholm-type integral equation for the unknown function $\phi$, and (6) is the related homogeneous equation. Hypothesis $\mathrm{H} 2$ on $K$, hypothesis $\mathrm{H} 1$ on the coefficients, and the fact that the singularity of $K$ is only logarithmic together imply that

$$
|G|<\frac{\text { constant }}{\left((z-r)^{2}+(t-s)^{2}\right)^{1 / 2}} .
$$

Fredholm has proved [4, p. $385 \mathrm{ff}$.] that for a kernel satisfying 
(7), the third iterated kernel is continuous, and hence (cf. [4]) either (5) has a unique solution $\phi$ which is bounded, or else (6) has a bounded solution not identically zero. In either case, $\phi$ bounded implies, by $[3$, p. 228] and the continuous differentiability of $f(z, t)$, that $\phi \in C^{1}$. Hence, in one case $u$ will satisfy $\left(1^{\prime}\right)$, and in the other it will satisfy $\left(1^{\prime \prime}\right)$. But this, together with the properties of $u$ which we proved earlier, establishes the two alternatives stated in the conclusion of the theorem, except for one matter: in case $\phi$ satisfies (6), we must show that $\phi \not \equiv 0$ implies $u \neq \equiv 0$. This however follows easily from the continuity of $\phi$ and the fact that $H(z, t ; r, s)$ is of unchanging sign so long as $(z, t)$ does not cross $W^{\prime}$. This completes the proof of the theorem.

Remark on the first alternative. In the following two cases, the first alternative is impossible, and the theorem becomes the usual type of boundary value problem existence theorem.

Case 1. Suppose that $T$ is so small that

$$
\iint_{T} \iint_{T} G_{2}^{2}(z, t ; r, s) d z d t d r d s<1,
$$

where $G_{2}$ is the iterate of $G$ and is known to belong to $L^{2}[3$, p. 282] in consequence of (7). In this case (6) can have only the solution $\phi \equiv 0$, as is easily shown by using Schwartz's inequality.

Case 2. In $\left(1^{\prime}\right)$, let $d=0$, and let

$$
c<-|e| l, \quad e<0
$$

where

$$
l=\max _{t} \int d z
$$

the integral being taken along a characteristic in $T$, from $W$ to $W$. Then, again, $\left(1^{\prime \prime}\right)$ can have no nontrivial solution with zero boundary values.

Proof. Let $u$ satisfy $\left(1^{\prime \prime}\right)$, and let $u_{z}=0$ on $W, u=0$ on $W^{\prime}$. Then, first, $u_{z}$ does not have a positive maximum in $T$ : let $M$ be a positive maximum, and consider the point where $u_{z}=M$. Then $\Delta\left(u_{z}\right) \leqq 0$ here, $u_{z s}=u_{z t}=0$, and we have

$$
\begin{aligned}
\Delta\left(u_{z}\right)+c M & =-e u, \\
c M & \geqq-e u, \\
-c M & \leqq e u, \\
-c M l & \leqq e M l,
\end{aligned}
$$




$$
-c \leqq e l,
$$

or

$$
c \geqq-e l,
$$

contradicting (8). So $u_{z}$ does not have a positive maximum in $T$. Therefore $u_{z} \leqq 0$ at every point of $T$, and hence $u \leqq 0$ in $T$.

Finally, $u_{z}$ can have no negative minimum in $T$. For, let $m<0$ be such a minimum, and consider the point where $u_{z}=m$. At this point,

$$
\begin{aligned}
\Delta\left(u_{z}\right)+c m & =-e u, \\
c m & \leqq-e u ;
\end{aligned}
$$

but $c<0, e<0, m<0, u \leqq 0$; hence $c m>0$, while $-e u \leqq 0$, and the smaller side of (9) is positive, while the larger side is nonpositive. So $u_{z}$ can have no negative minimum in $T$, and we conclude that $u_{z}=0$ in $T$.

But $u=0$ on $W^{\prime}$ then implies $u=0$ at every point of $T$. Therefore, in this case also, $\left(1^{\prime \prime}\right)$ can have no nontrivial solution with zero boundary values.

\section{BIBLIOGRAPHY}

1. L. Bieberbach, Theorie der Differentialgleichungen, New York, 1944.

2. R. Courant and D. Hilbert, Methoden der Mathematischen Physik, vol. 1, New York, 1943.

3. Idem, vol. 2.

4. I. Fredholm, Sur une classe d'équations fonctionnelles, Acta. Math. vol. 27 (1903) pp. 365-390.

5. J. Hadamard, Propriêtés d'une équation linéaire aux dérivées partielles du quatrième ordre, Tôhoku Math. J. vol. 37 (1933) pp. 133-150.

6. L. Lichtenstein, Neuere Entwicklung der Theorie Partieller Differentialgleichungen Zweiter Ordnung vom Elliptischen Typus, Encyklopädie der Mathematische Wissenschaften II 32, Leipzig, 1927, pp. 1277-1334.

7. O. Sjöstrand, Sur une equation aux dérivées partielles du type composite, Arkiv För Matematik, Astronomi Och Fysik vol. 25A, no. 21, pp. 1-11.

8. - Sur une équation aux dérivées partielles du type composite. Note 2, Arkiv För Matematik, Astronomi Och Fysik vol. 26A, no. 1 (1939) pp. 1-10.

Massachusetrs Institute of Technology 